\title{
Differential Pattern of cAMP Response Element-Binding Protein Activation in the Rat Brain after Conditioned Aversion as a Function of the Associative Process Engaged: Taste versus Context Association
}

\author{
Aline Desmedt, Shoshi Hazvi, and Yadin Dudai \\ Department of Neurobiology, The Weizmann Institute of Science, Rehovot 76100, Israel
}

\begin{abstract}
Ample data indicate that cAMP-response element-binding protein (CREB) is essential for the formation of long-term memory in various species and learning systems. This implies that activated CREB could delineate neuronal circuits that subserve items in memory, while leaving open the possibility that the specifics of CREB activation itself contribute to the specificity of the internal representation encoded by the relevant circuit. We describe here the differential activation of CREB in the rat brain as a function of two related yet distinct forms of aversive conditioning: conditioned taste aversion (CTA) and conditioned context aversion (CCA). We found that CTA induces strong CREB activation in the insular cortex (IC) and the lateral septum (LS), but not in the parietal cortex (PC) and the medial septum (MS). In contrast, CCA results in strong activation in the PC and MS, but not in the IC and LS. These findings are congruent with a model that links differential pattern of activity within the LS and the MS with the acquisition of elemental versus contextual conditioning and, more generally, with the notion that CREB activation delineates learning-dependent circuits as a function of the type of cognitive process engaged.
\end{abstract}

Key words: taste aversion; context aversion; insular cortex; parietal cortex; lateral septum; medial septum

\section{Introduction}

Certain molecular devices are assumed to fulfill universal roles in neuronal plasticity that subserves learning (Dudai, 1989, 2002; Martin et al., 2000). cAMP response element-binding protein (CREB) is a prominent example (Silva et al., 1998; Lamprecht, 1999; Taubenfeld et al., 1999; Kida et al., 2002). Phosphorylated, activated CREB binds to cAMP response element (CRE) in the promoter regions of early response genes, culminating in the induction of expression of additional genes whose products are thought to subserve synaptic remodeling (Sheng et al., 1990; Frank and Greenberg, 1994; Kwok et al., 1994). Ample evidence indicates that CREB is essential for long-term memory in multiple tasks in many species. Interference with CREB-mediated transcription attenuates long-term facilitation, a cellular analog of sensitization in Aplysia (Dash et al., 1990), whereas relief of CREB repression converts short- into long-term facilitation (Bartsch et al., 1995). In rodents, cued and contextual conditioning result in differential activation of CRE-mediated gene expression in the hippocampus (Impey et al., 1998), whereas CREmediated transcription in hippocampal slices, as well as increase

\footnotetext{
Received Feb. 20, 2003; revised April 16, 2003; accepted April 17, 2003.

This study was supported by a fellowship from the Weizmann Institute of Science (A.D.) and grants from the Human Frontier Science Foundation and the Volkswagen Stifftung (Y.D.). We thank Dr. D. E. Berman for helpful discussions and Dr. R. Eilam for technical assistance in the analysis of immunohistochemical data.

Correspondence should be addressed to Dr. Aline Desmedt, Department of Neurobiology, The Weizmann Institute of Science, Rehovot 76100, Israel. E-mail: aline.desmedt@weizmann.ac.il.

Copyright $\odot 2003$ Society for Neuroscience $\quad$ 0270-6474/03/236102-09\$15.00/0
}

in CREB phosphorylation in vivo, is induced by stimuli generating long-lasting long-term potentiation (LTP) (Impey et al., 1996; Schulz et al., 1999). Mutating the CREB gene in mice disrupts fear conditioning (Bourtchuladze et al., 1994), and targeted injection of CREB antisense into the hippocampus (Guzowski and McGaugh, 1997), or into the amygdala (Lamprecht et al., 1997), results in disruption of long-term spatial memory and of conditioned taste aversion (CTA), respectively.

The aforementioned involvement of CREB in long-lasting plasticity and memory suggests that it is a molecular building block, or syntactic neural plasticity device, whose contribution to the meaning [i.e., semantics at the representational level (Dudai, 1994, 2002)] is determined by the location of its activation. Seen this way, CREB could delineate circuits that subserve specific items in memory (Impey et al., 1998), converting the search for the engram into the search for the CREgram. The possibility, however, still remains that the specifics of CREB activation itself contribute to the specificity of internal representations encoded by the relevant circuits. It might be advantageous, as a step toward cross-level analysis of the relevance of CREB activation to representational specificity, to map and compare the differential patterns of CREB activation in multiple cognitive processes.

Toward this end, we describe in this study the differential activation of CREB in two related forms of aversive conditioning: classical CTA, an elemental association between a taste conditioned stimulus (CS) and the LiCl-induced malaise unconditioned stimulus (US), and conditioned context aversion (CCA), 
resulting from a prevalent association between the background training context and the same US. Specifically, we mapped CREB activation in the lateral septum (LS) and medial septum (MS), and the insular cortex (IC) and parietal cortex (PC). We focused on these brain regions, because they have been involved in elemental and contextual fear conditioning and/or in CTA. Whereas the IC and the septum have been shown to be involved in CTA (Siegel, 1976; Yamamoto and Fujimoto, 1991; Rosenblum et al., 1993; Yamamoto, 1994; Naor and Dudai, 1996), the LS and MS have been implicated in setting the imbalance between elemental and contextual fear conditioning (Garcia and Jaffard, 1996; Desmedt et al., 1998, 1999), and CREB activation within the PC has been related to contextual fear conditioning (Stanciu et al., 2001). We thus hypothesized differential CREB activation in these four brain regions after aversive conditioning, depending on whether the prevalent conditioned association is based on the taste CS (CTA) or on the training background context (CCA). Our findings are congruent with this hypothesis and, furthermore, support the notion that CREB activation delineates circuits that encode the most salient facet of a learning experience.

\section{Materials and Methods}

Subjects. Rats (Wistar males; 3 months of age; 250-300 gm; Weizmann Institute of Science) were housed individually in opaque plastic boxes $(35 \times 30 \times 15 \mathrm{~cm}$ high $) 7 \mathrm{~d}$ before the experiment $\left(22 \pm 2^{\circ} \mathrm{C}\right)$, under a 12 $\mathrm{hr}$ artificial light/dark cycle and with ad libitum access to food and water, unless otherwise indicated. Caging, handling, and experimentation were in following with the rules of the Weizmann Institute Animal Experiments Committee.

Reagents. Anti-phosphorylated CREB (pCREB) antibody was from Upstate Biotechnology (Lake Placid, NY). Anti-CREB antibody was from Cell Signaling Technology (Beverly, MA). Biotinylated goat anti-rabbit antibody (BA-1000) and avidin DH-biotinylated horseradish peroxidase (HRP) H reagents were from Elite (Vectastain; Vector Laboratories, Burlingame, CA). PK-3' -diaminobenzidine tetrahydrochloride (DAB) (Sigma Fast tablets; D-4293) was from Sigma (St. Louis, MO). All of the other chemicals were of the highest grade available.

Apparatus. Conditioned aversion took place in a clear Plexiglas box $(50 \times 15 \times 15 \mathrm{~cm}$ high $)$ allowing perception of the visuospatial cues in the experimental room and defined as the conditioning context. A second box $(35 \times 30 \times 15 \mathrm{~cm}$ high $)$, made of opaque plastic and similar to animal's home cage, served as neutral context and was used for both habituation to liquid consumption and the behavioral tests (see below), either alone (measure of taste aversion), or in conjunction with the previous box (measure of context avoidance).

Behavioral procedure. In CTA, organisms learn to avoid a novel tastant if its ingestion is followed by transient malaise (Garcia et al., 1966; Bures et al., 1998). Here, we compared CTA with a related learning protocol designed to result in aversion to the training context (CCA). CTA was obtained by pairing the taste CS $(0.1 \% \mathrm{w} / \mathrm{v}$ saccharin) with the malaiseinducing agent $\mathrm{LiCl}$ intraperitoneally $(0.15 \mathrm{M} ; 2 \%$ body weight; US) in a classical forward-conditioning protocol. CCA was obtained by presenting the same CS after termination of the malaise (i.e., in a backwardconditioning protocol with regard to the above CS and US). This is ineffective in associating the taste CS with the US but is supposed to promote the association of the US with the conditioning context at the time of its experience. Hence, both procedures were counterbalanced with regard to the stimuli used and the extent of exposure to these stimuli yet differed in the associations generated.

Rats were water deprived for $24 \mathrm{hr}$ and then pretrained over $3 \mathrm{~d}$ to get their daily water ration within $10 \mathrm{~min}$ per day from two pipettes, each containing $10 \mathrm{ml}$. This pretraining took place in the neutral context. On day 4 (conditioning day), the rats were exposed to the conditioning context and were submitted to one of the following procedures: they received saccharin instead of water either $30 \mathrm{~min}$ before being injected with $\mathrm{LiCl}$ intraperitoneally (defined here as the CTA group) or $155 \mathrm{~min}$ after $\mathrm{LiCl}$ injection (defined here as the CCA group). Whatever the behavioral procedure used, all of the animals remained in the conditioning context for a total period of $210 \mathrm{~min}$. The behavioral responses to $\mathrm{LiCl}$ injection [i.e., lying on the belly (LOB) (Meachum and Bernstein, 1990) and rearing (Parker et al., 1982)] were monitored at intervals of $30 \mathrm{~min}$ until the end of training, and saccharin consumption was recorded. As described previously (Lamprecht et al., 1997), the malaise induced by the negative reinforcer dissipated within $<2 \mathrm{hr}$, as assessed by the disappearance of LOB (prominent behavioral index of malaise) $2 \mathrm{hr}$ after $\mathrm{LiCl}$ injection. Three rats were excluded from additional analysis because of an unclear malaise response. Two control groups were subjected to the same procedure with presentation of saccharin either $10 \mathrm{~min}$ [saccharin-atbeginning (SACBEG) group] or $195 \mathrm{~min}$ [saccharin-at-end (SACEND) group] after being placed in the conditioning context (exactly as in the CTA and CCA groups, respectively) but did not receive $\mathrm{LiCl}$ injection. These two control groups were intended to control for any effect that might result from the difference in timing of saccharin presentation relative to the end of training in the CTA and CCA groups, respectively. Each animal was then returned to its home cage in the animal room. Some of the animals $(n=56)$ were killed (as detailed below) 30 min later for immunohistochemistry of pCREB, whereas the remaining rats $(n=$ 41) remained in the animal room for $24 \mathrm{hr}$ and were further submitted to behavioral testing. In an additional group (NAIVE), rats were taken directly from their home cages and killed (as detailed below) for immunohistochemistry analysis.

On day 5, animals were first tested for aversion to saccharin. They were maintained in the neutral context and were presented with an array of six pipettes, three containing $5 \mathrm{ml}$ of saccharin and three containing $5 \mathrm{ml}$ of water. The aversion index (AI) was defined as follows: ([milliliters of water/(milliliters of water + milliliters of saccharin $)] \times 100)$ consumed in the test (Rosenblum et al., 1993) (i.e., AI = 50 is equal preference level, and the higher the AI, the more the rats prefer water to saccharin). Animals were then replaced in their home cage. Two hours later, the same animals were tested for context aversion: from a starting compartment $(45 \times 15 \times 15 \mathrm{~cm}$ high) given access to the neutral and to the conditioning contexts, they were allowed to enter in both contexts for $30 \mathrm{~min}$ (Fig. 1 ). The time spent in each context was recorded. Because animals were free to enter each of the three compartments throughout the session, 33\% is chance level. We reasoned that the less the time spent in the conditioning context, the more the rats have associated the aversive experience (LiCl-induced malaise) with that context (Fig. 1).

Immunohistochemistry and image analysis. Animals were deeply anesthetized with equitesin $(4.3 \mathrm{ml} / \mathrm{kg}$, i.p.), and transcardially perfused by gravity drip infusion with PBS (heparin, $4 \mathrm{U} / \mathrm{ml}$ ) followed by cold, fresh $2.5 \%$ paraformaldehyde and $5 \%$ sucrose in PBS, $\mathrm{pH}$ 7.4. The brain was postfixed for $48 \mathrm{hr}$ in $1 \%$ paraformaldehyde and 30\% sucrose in PBS at $4^{\circ} \mathrm{C}$. Four rats from the CCA group and two rats from the control SACBEG group were excluded from additional analysis because of poor perfusion quality. Postfixed brains were sectioned coronally on a freezing microtome at a thickness of $50 \mu \mathrm{m}$. Sections were collected in 24-well plates containing PBS and azide $(0.01 \%)$. Sections were then washed three times ( 5 min each) in PBS and immersed for $45 \mathrm{~min}$ in $49.5 \%$ methanol, $49.5 \% \mathrm{PBS}$, and $0.9 \% \mathrm{H}_{2} \mathrm{O}_{2}$, followed by washing five times (5 min each) with PBS and immersion for $20 \mathrm{~min}$ in $0.15 \mathrm{M}$ glycine in PBS, $\mathrm{pH}$ 7.4. The sections were then blocked with $20 \%$ normal goat serum (NGS) in PBS and $0.5 \%$ Triton X-100 for $2.5 \mathrm{hr}$, followed by $16 \mathrm{hr}$ incubation at room temperature with the anti-pCREB antibody (1:500) or anti-CREB (1:400) and 2\% NGS in PBS. The sections were then washed (three times; 5 min each) with PBS and incubated with biotinylated goat anti-rabbit antibody (1:200) and 2\% NGS in PBS for $1.5 \mathrm{hr}$ at room temperature, followed by three additional PBS washings (5 min each). Finally, avidin DH-biotinylated horseradish peroxidase $\mathrm{H}$ complex (1:50) was added for $1.5 \mathrm{hr}$. The sections were washed once with PBS and two times with $50 \mathrm{~mm}$ Tris-Cl buffer, $\mathrm{pH}$ 7.5. Peroxidase activity was determined by reaction with a mixture containing $0.7 \mathrm{mg} / \mathrm{ml} \mathrm{DAB}$ and 2 $\mathrm{mg} / \mathrm{ml}$ urea- $\mathrm{H}_{2} \mathrm{O}_{2}$ in $60 \mathrm{~mm}$ Tris-Cl. The sections were washed three times with Tris buffer and immersed for $3 \mathrm{~min}$ in cobalt-enhanced DAB (1:4). They were then mounted on slides, dried, dehydrated by successive 
rinses of 70, 95, and 100\% ethanol (two times) and $100 \%$ xylene (two times), and covered with Permount.

Because pCREB immunoreactivity was found to be exclusively nuclear (Hall et al., 2001), nuclear counting was the preferred method of analysis. We prepared computer images of photomicrographs of sections by using a video camera (DXM1200; Nikon, Melville, NY) attached to a Nikon 800 microscope. Frames were recorded by using the software Act 1 (Nikon) and transferred as JPEG files to Image-Pro Plus 4.1 image analysis software (Media Cybernetics, Silver Spring, MD) for processing. Sections were scored using a region of interest (ROI) specific for each brain area. For LS, MS and IC, four measurements for each region were made from four different sections per animal and averaged. All of the sections quantified were between bregma $+1 \mathrm{~mm}$ and bregma $-0.26 \mathrm{~mm}$ (Paxinos and Watson, 1986). Specifically, for the septal region, the LS sample was focused on the dorsal part of the region, and within the MS, the analysis was focused on the medial septal nucleus and dorsal part of the vertical limb diagonal band. Within the IC, disgranular and granular areas were targeted. For the PC, six measurements were made from six different sections per animal and averaged. All of these sections were between bregma $+1 \mathrm{~mm}$ and bregma $-3.30 \mathrm{~mm}$, and the analysis was focused on the primary area (somatosensory cortex).

The threshold of positive immunoreactive staining was set by the experimenter and was determined by eye as cellular staining darker than the surrounding neuropil. The computer automatically counted all of the positive targets in the ROI, excluding those with an area $<5 \mu \mathrm{m}^{2}$ to reject noncellular irregularities in the background staining. Results were expressed as the number of PCREB-positive nuclei per $0.5 \mathrm{~mm}^{2}$ for each region. At all of the stages, the experimenter was blind to the experimental groupings. The same procedure was followed for immunohistochemistry of total CREB (Lamprecht et al., 1997).

Statistics. Statistical analysis of behavioral and immunohistochemical data were performed using ANOVA and $t$ test (Systat, Evanston, IL) to make individual comparisons between groups and means comparisons within groups, with an $\alpha$ level of 0.05 .

\section{Results}

The CTA and CCA groups displayed preferential conditioned aversion to either the taste or the conditioning context, respectively

Conditioned aversion to saccharin was different among the four groups, with the CTA group being the only one to display significant aversion (Fig. 2A). One-way ANOVA performed on these data with the training procedure as the between-groups factor indicated a significant difference among groups $\left(F_{(3,37)}=42.17\right.$; $p<0.001)$. Post hoc analysis revealed that the CTA group displayed a significantly stronger aversion to saccharin compared with the CCA, SACBEG, and SACEND control groups with all of the $p$ values $<0.001$. All of these latter three groups did not significantly differ from each others $\left(F_{(2,24)}=0.64\right.$; NS). Moreover, the CTA group was the only one to display a significant AI compared with $50 \%$ equal preference $(p<0.001)$. The three other groups displayed a close-to-chance performance with significant preference for saccharin in the SACBEG group $(p=0.015)$.

In the conditioned context avoidance test (Fig. $2 B$ ), the time spent in each context (i.e., conditioning vs neutral) differed ac- $\underline{\text { Aversive conditioning }}$

$\underline{\text { Tests }}$

Conditioned taste aversion (taste CS-US pairing, CTA)

$(2 \mathrm{~h})$

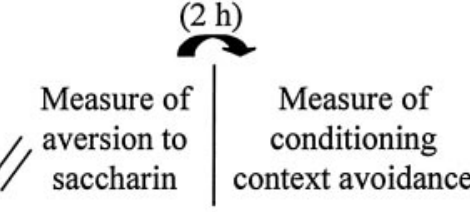

LiCl (Day 4)

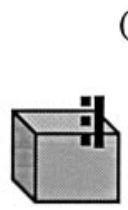

conditioning context

(Day 5)

Time spent?

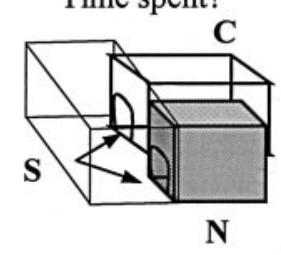
were the only ones to spend more time in the neutral context than in the conditioning context. They spent more time in the neutral context than either the CTA $(p=0.04)$, SACEND $(p=0.02)$, or SACBEG $(p=0.03)$ groups. They also spent less time in the conditioning context than either rats in the CTA $(p=0.04)$ or the SACEND ( $p=0.04)$ groups. This led to a significant contextby-training procedure interaction when the two experimental groups (i.e., CTA and CCA) were considered $\left(F_{(1,25)}=5.02 ; p=\right.$ $0.03)$. Moreover, animals in the CCA group spent more time in the neutral context than in the conditioning context $(p=0.02)$, a difference that was not observed in any of the three other groups (all of the $p$ values were $>0.05$ ). Finally, with respect to chance level (33\%), whereas the CCA rats spent more time in the neutral context $(p=0.015)$ and less time in the conditioning context $(p=0.04)$, none of the three other groups, which did not differ from each other $\left(F_{(2,25)}=1.54 ; \mathrm{NS}\right)$, displayed such differences (all of the $p$ values were $>0.05$ ).

Taste and context conditioning each resulted in a different pattern of CREB phosphorylation in the septal region

In the LS, specifically within the dorsal LS (Fig. 3A), a differential activation of CREB was observed according to the type of training procedure (Fig. 4, top). In particular, in the CTA group, the number of positive immunoreactive nuclei was much higher than in rats from any of the other groups. ANOVA performed on these data with training condition as between-groups factor indicated a significant effect of training $\left(F_{(4,49)}=6.48 ; p<0.001\right)$. Specifically, the number of positive immunoreactive nuclei in the CTA group was higher than in the CCA $(p=0.04)$, SACBEG $(p=0.012)$, SACEND $(p=0.006)$, or NAIVE $(p=0.012)$ groups. These between-groups differences were observed whichever brain hemisphere was considered (hemisphere $\times$ training 

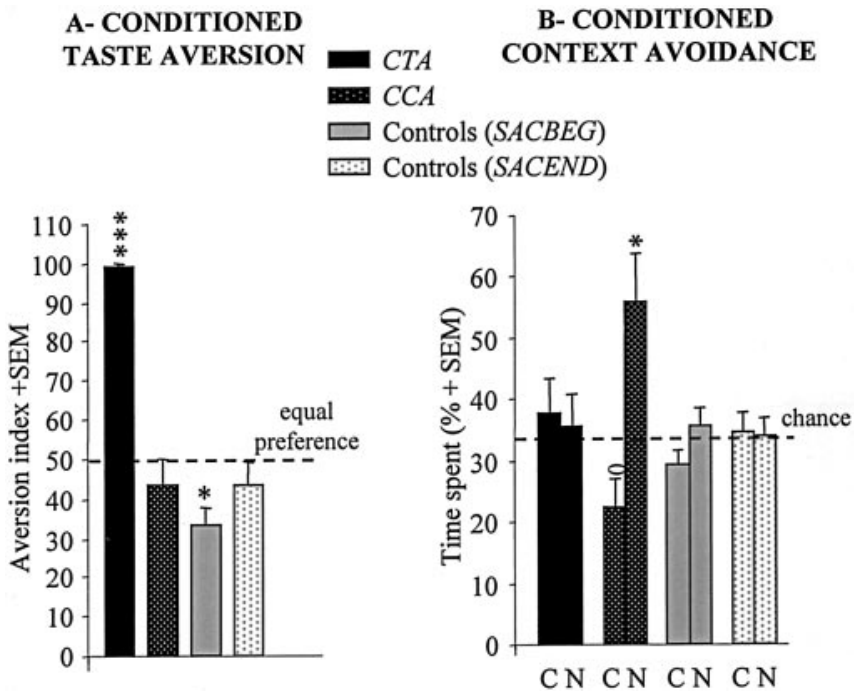

Figure 2. CTA versus CCA. Amplitude of the Al to saccharin (mean + SEM) $(A)$ and time spent (mean + SEM) in the conditioning $(C)$ and neutral $(N)$ contexts $(B)$ for rats in the CTA group $(n=14)$ or the CCA group $(n=13)$, and for the SACBEG $(n=6)$ and SACEND $(n=8)$ groups. In $A,{ }^{* * *}$ statistically significant (CTA group vs each of the other groups, $p<0.005$ ); in $B,{ }^{*}$ significantly different from each of the other groups and from chance level $(p<0.05)$; ${ }^{\circ}$ statistically different within the CCA group ( $\left.p=0.02\right)$. Dotted horizontal lines represent equal preference for water and saccharin in the taste choice situation $(A)$ and chance level for the choice between the three compartments in the contextual test situation $(B)$.

interaction, all of the $p$ values were $>0.08)$. Otherwise, these latter four groups did not significantly differ from each other $\left(F_{(3,39)}=\right.$ 1.59 ; NS).

In contrast, within the MS (Fig. 3B), although differential activation of CREB was also noticed according to the type of training procedure (Fig. 4, bottom), the CCA group had more positive immunoreactive nuclei than rats in each of the other groups. In particular, the CCA group displayed higher pCREB level in this region than rats in the CTA $\left(F_{(1,17)}=5.59 ; p=0.03\right)$, SACEND $\left(F_{(1,20)}=5.45 ; p=0.03\right), \operatorname{SACBEG}\left(F_{(1,18)}=5.36 ; p=0.03\right)$, or $\operatorname{NAIVE}\left(F_{(1,14)}=12.55 ; p=0.003\right)$ groups, which otherwise did not significantly differ from each other $\left(F_{(3,41)}=0.50\right.$; NS).

Taste and context conditioning each resulted in a different pattern of CREB phosphorylation in the IC and PC

Differential CREB activation was observed within the IC as a function of the type of training procedure (Fig. $3 C$ ). Rats in the CTA group displayed in this brain region a number of positive immunoreactive nuclei that was higher than that found in each of the four other groups. Interestingly, this difference was restricted to the right hemisphere (Fig. 5). A two-way ANOVA with brain hemisphere (left vs right) as the within-subjects factor and training procedure as the between-groups factor revealed a significant effect of training $\left(F_{(4,49)}=3.41 ; p=0.015\right)$ and of hemisphere $\left(F_{(1,49)}=17.76 ; p<0.001\right)$ with a significant hemisphere-bytraining interaction $\left(F_{(4,49)}=6.88 ; p<0.001\right)$. One-way ANOVA revealed that the between-groups differences were restricted to the right IC $\left(F_{(4,49)}=5.49 ; p=0.001\right)$. No significant differences were found within the left hemisphere $\left(F_{(4,49)}=1.76\right.$; NS). Specifically, the CTA group displayed a higher number of positive immunoreactive nuclei in the right IC than either the CCA $(p=0.03)$, SACBEG $(p=0.01)$, SACEND $(p=0.01)$, or NAIVE $(p=0.018)$ groups, which otherwise did not significantly differ from each other $\left(F_{(3,39)}=0.61\right.$; NS). Moreover, the CTA group displayed significant lateralization (i.e., much more posi-
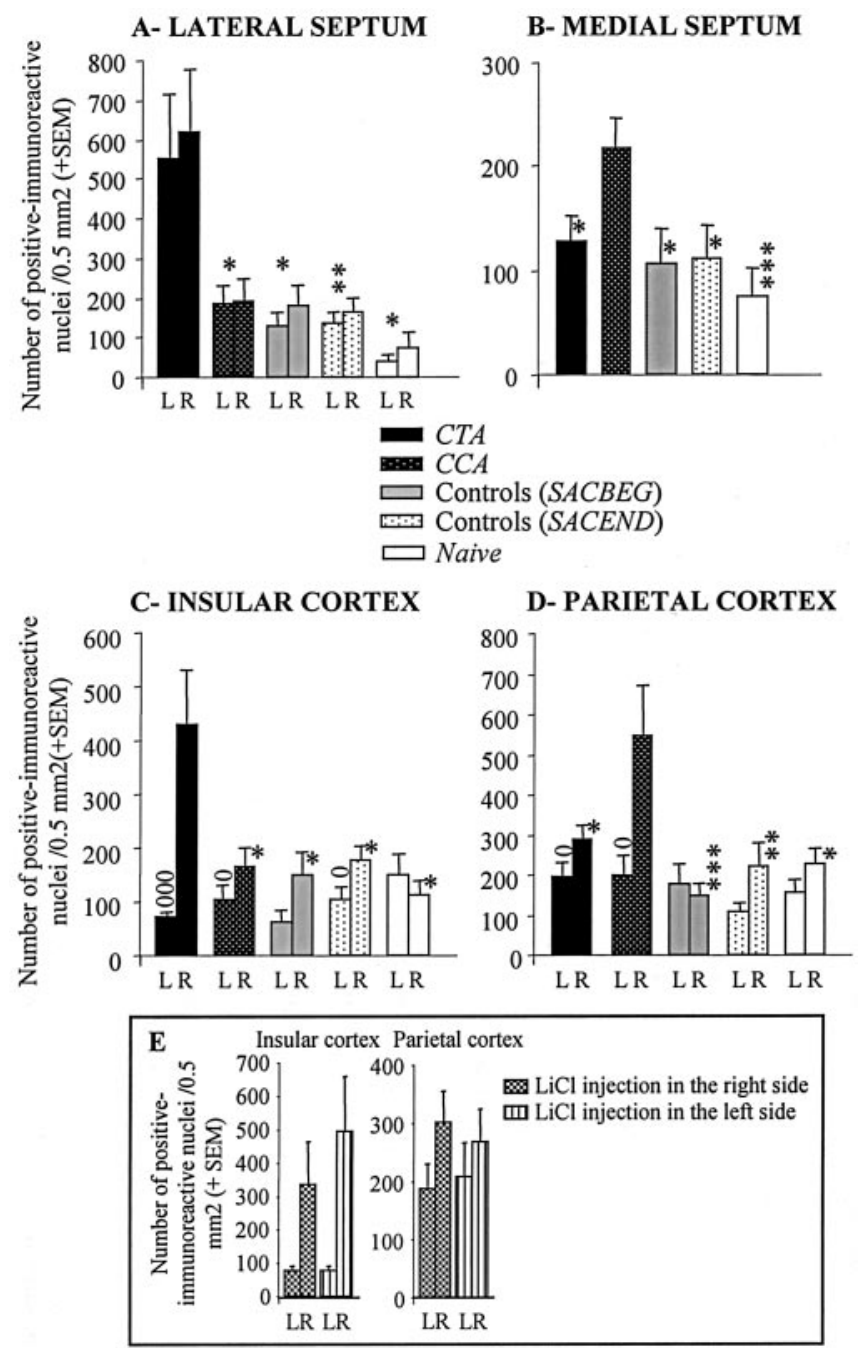

Figure 3. Number of positive pCREB-immunoreactive nuclei ( + SEM) within the $L S(A)$, MS $(B), I C(C)$, and PC (D) in the CTA $(n=13), C C A(n=9), \operatorname{SACBEG}(n=12), \operatorname{SACEND}(n=14)$, and NAIVE animal $(n=8)$ groups. For each group, results obtained from each hemisphere [left $(\mathrm{L})$; right (R)] are presented for the $L S, I C$, and $P C$. E, Results obtained within the two cortical areas when the CTA group was subdivided into rats that received LiCl injection intraperitoneally into the right side of the body and rats that received this injection into the left side. ${ }^{* *} p<$ $0.005 ;{ }^{* *} p<0.01 ;{ }^{*} p<0.05$. ${ }^{\circ}$ Significant difference between the left and the right hemispheres, $p<0.05 ;{ }^{\circ 000} p<0.005$.

tive immunoreactive nuclei in the right hemisphere compared with the left one; $p=0.005)$. Although rats in both the CCA and the SACEND groups also displayed such lateralization $(p=$ 0.012 and $p=0.04$, respectively), significant hemisphere-bytraining procedure interaction performed on pooled data from the CTA group and from each of the other groups (all of the $p$ values were $<0.02$ ) indicated more pronounced lateralization in the CTA group.

Differential CREB activation was also observed within the PC as a function of the type of training procedure (Fig. 3D). In contrast to the IC, the highest number of positive immunoreactive nuclei was found in the CCA group. As in the previous analysis (Fig. 3C), this between-groups difference was restricted to the right brain hemisphere (Fig. 6). A two-way ANOVA with hemisphere as the within-subject factor and training procedure as the between-groups factor indicated a significant effect of the training procedure $\left(F_{(4,46)}=4.32 ; p=0.005\right)$ and of hemisphere $\left(F_{(1,46)}=18.21 ; p<0.001\right)$ with a significant hemisphere-by- 
training interaction $\left(F_{(4,46)}=4.32 ; p=\right.$ 0.005). In particular, in the CCA group, the number of positive immunoreactive nuclei in the right PC was significantly higher than either in CTA $(p=0.03)$, SACEND $(p=0.01)$, SACBEG $(p=$ $0.001)$, or NAIVE $(p=0.02)$ groups, which otherwise did not significantly differ from each other $\left(F_{(3,40)}=1.43\right.$; NS) whichever brain hemisphere was considered (all of the $p$ values were $>0.1$ ). Moreover, rats in the CCA group displayed significant lateralization (i.e., higher number of immunoreactive nuclei in the right PC compared with the left one) $(p=0.03)$. Although rats in the CTA group also displayed such a lateralization ( $p=0.037)$, a significant hemisphere-by-training procedure interaction performed on pooled data from the two experimental groups $(p=0.03)$ indicated a more pronounced lateralization in CCA rats. No significant difference in the level of positive immunoreactive nuclei between the right and the left PC was found for any of the three other groups (all of the $p$ values were $>0.05$ ).

The above differences in CREB phosphorylation between the experimental groups, both in the septal region and in the IC and PC, cannot be accounted for by the effect of taste CS, malaise US, or context exposure per se, insofar as all of these stimuli were present equally in all of the training procedures. One could still argue that the difference in the timing of saccharin presentation accounts for such differences in CREB phosphorylation. This, however, is unlikely, because none of the differences observed between the two experimental groups, CTA and CCA, was found between the two control groups, SACBEG (presented with saccharin at the beginning of training as in the CTA group) and SACEND (presented with saccharin at the end of training as in the CCA group); all of the $p$ values were $>0.05$, whichever brain area was considered. Together, these results strongly suggest that beyond learning per se, the type of conditioned association performed (i.e., elemental taste CS-US or context-US association) accounts for such differential patterns of CREB activation.

It is noteworthy that, in both the IC and the PC, we found stronger CREB phosphorylation in the right hemisphere $(p<$ 0.001 in both cases). Post hoc analysis indicated that the difference in the number of positive immunoreactive nuclei between the left and the right brain hemispheres was more pronounced in the CTA group (IC) (Fig. 5) and in the CCA group (PC) (Fig. 6) when compared with all of the other groups. While being amplified by learning according to the type of conditioned association performed, this phenomenon was observed irrespective of the $\mathrm{LiCl}$ injection side (i.e., right or left side of the body) (Fig. 3E), which-

CTA

CCA
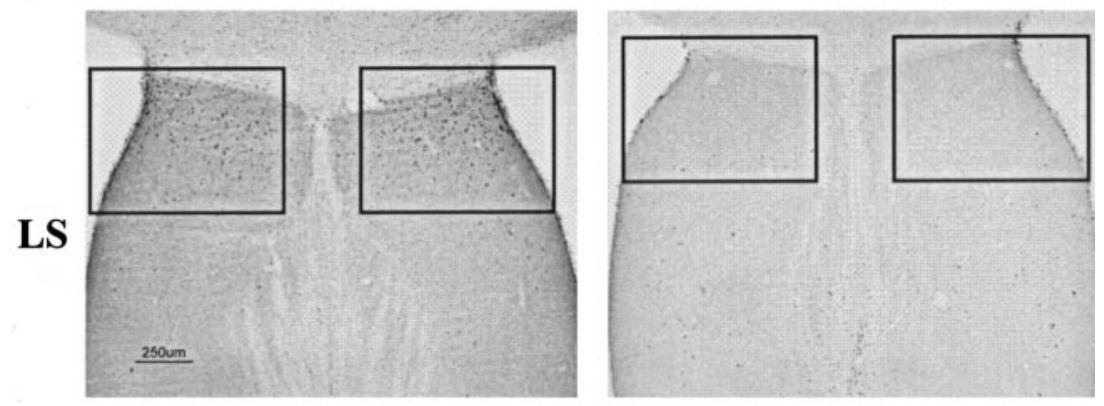

Figure 4. Representative photomicrographs depicting pCREB levels within the LS (top) and the MS (bottom) in rats from the CTA or the CCA groups, respectively. The frames define the regions of interest.

\section{INSULAR CORTEX}

\section{Left hemisphere}
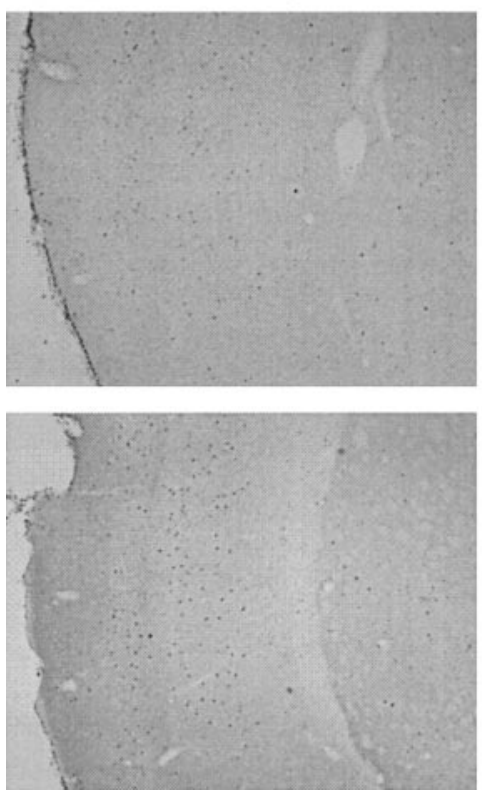

Figure 5. Representative photomicrographs depicting pCREB levels within the left and right IC in rats from the CTA or CCA groups, respectively.

ever brain structure was considered [all of the $p$ values were $>0.05$; in particular, there was no effect of the injection side on pCREB levels within the IC $(p>0.6)$ or the PC $(p>0.9)$ and no differential lateralization between the two groups whichever cortical brain region was considered (all of the $p$ values were $>0.5$ )] This suggests that the observed lateralization, which was not pursued further in this study, was not attributable to an injection side-related perceptual or visceral effect. 


\section{PARIETAL CORTEX}

\section{Left hemisphere}
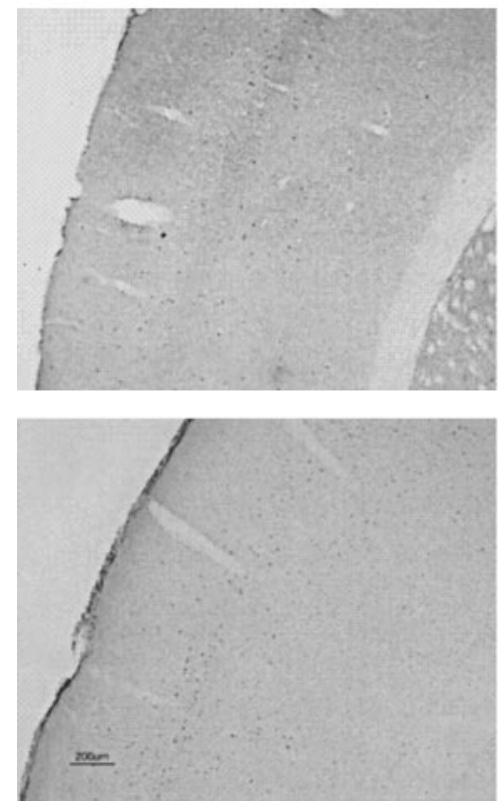

Figure 6. Representative photomicrographs depicting $p C R E B$ levels in the left and right PC in rats from the CTA or the CCA groups, respectively.

\section{On the contribution of phosphorylation versus expression of CREB}

Modulation of pCREB levels could result either from differential activation of CREB or from differential expression of CREB. We established that the differences observed in our study in the levels of immunoreactive nuclei between the experimental groups were mostly attributable to changes in CREB phosphorylation rather than in CREB expression. We replicated the analysis using antiCREB antibody and found similar levels of immunoreactive nuclei in the experimental and NAIVE groups, whichever brain structure was considered (Fig. 7) (all of the $p$ values were $>0.6$ ). This analysis also revealed, however, that compared with the left hemisphere, animals in the CCA group displayed significantly higher number of immunoreactive nuclei in the right $\mathrm{PC}$ ( $p=$ 0.003 ), whereas animals in the CTA group displayed significantly higher number of immunoreactive nuclei in the right IC ( $p=$ 0.04). Although tendency for such lateralization within the PC was observed in the CTA and the NAIVE groups, the differences between the left and the right hemispheres did not reach statistical significance (all of the $p$ values were $>0.05$ ) (Fig. $7 B$ ). This indicated that the high lateralization observed in the PC and in the IC for the CCA group and the CTA group, respectively, might also involve lateralization in CREB expression.

\section{Discussion}

The main finding of this report is that two different aversive learning situations, one involving elemental (taste) conditioned association and the other involving contextual association, result in differential CREB activation in the septal region and in two different cortical areas. CTA results in strong CREB activation in the LS and the IC, but not in the MS and the PC. In contrast, a CS-US backward-conditioning procedure, culminating in CCA as expected on the basis of competitive models of associative learning (Marlin, 1981), results in strong activation in the MS and the PC, but not in the LS and the IC. Previous studies have re- ported differential c-Fos induction (Spray et al., 2000) and glutamate release (Miranda et al., 2002) in CTA that are dependent on the brain region or the CTA protocol, respectively. The present study focuses on differential CREB activation as a function of both the brain region and the type of associative process, in an attempt to better understand the link between a universal molecular plasticity device and specific cognitive processes.

The type-of-association dependency of CREB activation in the septum is in line with a model suggesting that high activity in the dorsal LS, combined with low activity in the MS, is required for elemental conditioning, whereas the opposite pattern of activity is required for contextbased conditioning (Desmedt et al., 1999). Whereas contextual fear conditioning is associated with decreased hippocampal (HPC)-LS synaptic transmission, elemental fear conditioning (i.e., tone CS-footshock US pairing) is associated with either no change (Garcia and Jaffard, 1996; Desmedt et al., 1998) or increase in this transmission (Desmedt et al., 2003), depending on the strength of the elemental association. Moreover, experimentally induced increases in HPC-LS neurotransmission have been associated with both an increase in elemental fear conditioning and a decrease in contextual conditioning (Vouimba et al., 1998; Desmedt et al., 1999), whereas LS lesions specifically potentiate conditioning to context (Sparks and LeDoux, 1995). In contrast, an increase in acetylcholine release in the MS-HPC pathway, which is dependent on depression in GABAergic release from the LS (Giovannini et al., 1994), has been related to spatial learning and contextual conditioning tasks (Marighetto et al., 1994; NailBoucherie et al., 2000). Together, these data suggest a critical role of the septum in regulating the imbalance between elemental and contextual associations. Congruent with this, the present findings suggest that CREB subserves this differential requirement of LS and MS in conditioning.

The IC fulfils a key role in taste learning and CTA (Rosenblum et al., 1993, 1997; Berman et al., 1998, 2000), yet pCREB increase in the IC has so far been related only to the immediate effect of intraperitoneal $\mathrm{LiCl}$ injection (Swank, 2000). In the present study, the CCA group received $\mathrm{LiCl}$ intraperioneal injection yet did not display such increased insular pCREB level. It is the taste CS-malaise US pairing that specifically leads to CREB activation in the IC. The fact that, in Swank (2000), animals were killed 30 min after $\mathrm{LiCl}$ or $\mathrm{NaCl}$ intraperitoneal injection (i.e., when animals expressed either intense visceral malaise or no malaise at all), whereas, in our experiment, all of the rats were killed after the expression of malaise, might account for this discrepancy. Moreover, given the biphasic dynamic of CREB phosphorylation after LTP induction (Schulz et al., 1999) or aversive conditioning (Stanciu et al., 2001), comparison of studies using different time points of killing deserves caution. Nevertheless, our data show that CREB activation in the IC cannot be attributable to an aversive LiCl-induced effect alone but is rather related, in a latter phase of learning, to the establishment of elemental taste-malaise association. Interestingly, lesions of the posterior extension of the 


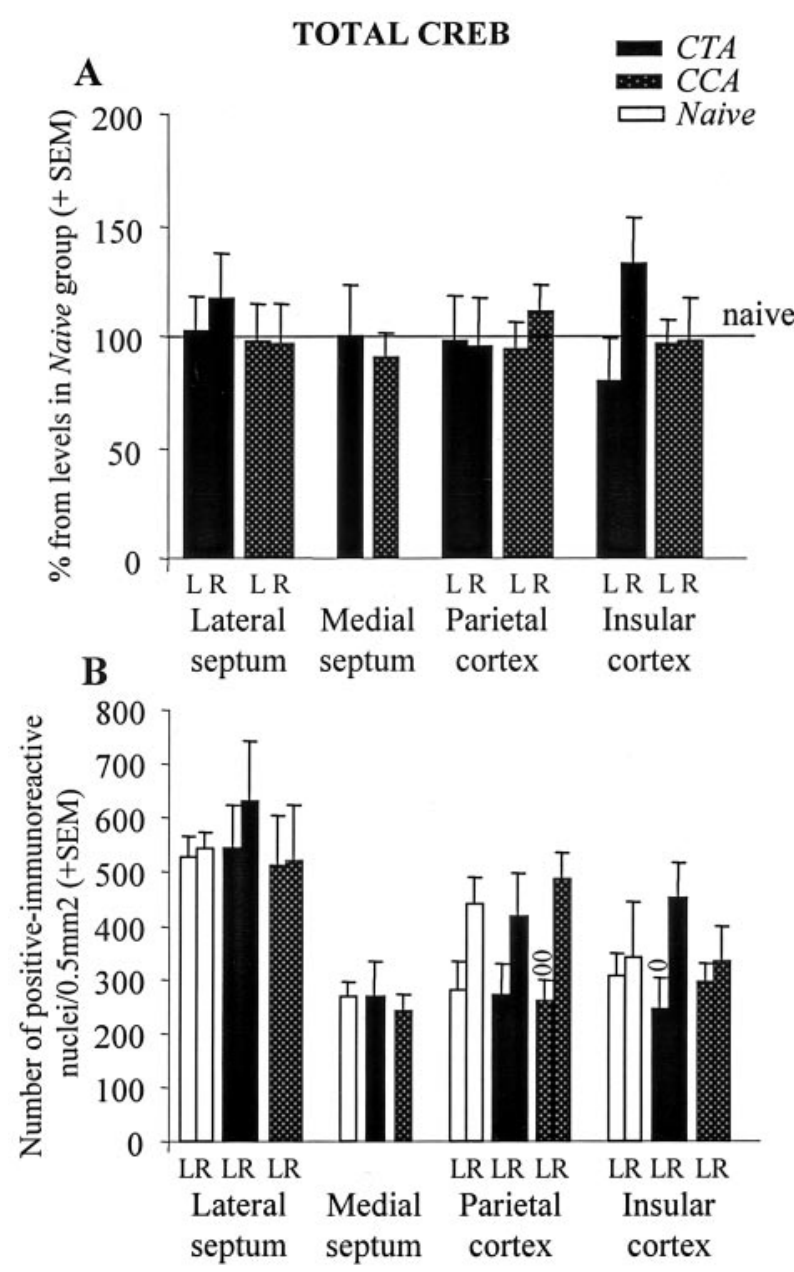

Figure 7. A, Total CREB levels (mean + SEM) in the experimental groups as percentages of CREB levels in the NAIVE group. Horizontal line represents the level of Total CREB in NAIVE group. $B$, Total CREB levels (mean + SEM) within the LS, MS, PC, and IC in the NAIVE, CTA, and CCA groups, respectively. ${ }^{\circ}$ Significant difference between the left and right hemispheres, $p<0.05 ;{ }^{\circ 0} p<0.01$. L, Left; $\mathrm{R}$, right.

intralaminar complex and caudal IC result in attenuation of elemental tone conditioning, while sparing contextual conditioning (Brunzell and Kim, 2001). This reinforces the possibility of a prevailing requirement of the IC in elemental conditioning as opposed to contextual conditioning.

The observation that the highest pCREB level within the PC is detected in the CCA group is congruent with data showing that CREB phosphorylation in PC (but also in the hippocampus and basolateral and central amygdala) correlates with contextual fear conditioning (Stanciu et al., 2001). The observation that the highest pCREB levels immediately posttraining are related to the footshock US, whereas pCREB levels at 3-6 hr posttraining are selectively related to contextual conditioning, led Stanciu et al. (2001) to suggest that the early phase of pCREB increase is related to stress, whereas the late phase is related to memory consolidation. The early stress-related peak fits the aforementioned data of Swank (2000). In our study, animals were killed $>3 \mathrm{hr}$ after $\mathrm{LiCl}$ injection; thus, the parietal pCREB increase selective to the CCA group supports a role of CREB in the $\mathrm{PC}$ in consolidation of contextual, but not elemental, association.

From a systems point of view, the mere finding that identified areas are specifically coactivated in these tasks suggests that we are observing fragments of circuits subserving in each case the rele- vant association. What is already known about the role of these areas in learning supports such assumption. The LS and IC have been repeatedly implicated in CTA (Siegel, 1976; Rosenblum et al., 1993; Yamamoto, 1994; Yamamoto et al., 1995; Naor and Dudai, 1996) and are both involved in a neural network that convey visceral stimuli to the diffuse thalamocortical relay system (Otake et al., 1994), which might contribute to CTA. Moreover, lesions to the IC impair both flavor-illness and tone-shock conditioning (Hankins et al., 1974), and both IC and LS have been implicated in controlling elemental CS-US association (Yamamoto et al., 1994; Desmedt et al., 1999). All in all, these data support the suggestion that activation of these two brain regions subserves taste-malaise association.

Our data are also congruent with previous reports indicating a role for the MS and PC in context-based aversion (Radulovic et al., 1998; Desmedt et al., 1999; Stanciu et al., 2001). These structures are parts of two cholinergic systems (McKinney et al., 1983; Mesulam et al., 1983; Bucci et al., 1999), which have been implicated in spatial and attentional processing (Olton et al., 1991; Muir et al., 1994; Chiba et al., 1995, 1999; Bucci et al., 1998; Baxter and Chiba, 1999; Wrenn et al., 1999; Nakamura et al., 2002). Whereas the PC is required for increased attentional processing of conditioned stimuli (Bucci et al., 1998) and for detection of spatial novelty (Thinus-Blanc et al., 1996), lesions to the MS impaired spatial working memory in the radial-maze task (Miyamoto et al., 1987; Wrenn et al., 1999) and spatial memory in the water maze (Miyamoto et al., 1987; Hagan et al., 1988; Kelsey and Laudry, 1988). Specifically, lesions to the MS reduce the capacity to rely on a representation of the relationship between distant contextual cues, while promoting a pure guidance strategy based on the most salient proximal cue (Brandner and Schenk, 1998). All of this suggests that both the MS and PC might contribute to place learning, an assumption that supports a role in CCA as well.

Because the interplay among LS and MS activity has been considered to be a sliding switch that contributes to the imbalance between elemental and contextual conditioning (Desmedt et al., 1999), the present findings raise the possibility that inactivation of either the LS or the MS would differentially alter CREB activation within the IC and the PC and result, thereby, in impairment of CTA or CCA, respectively.

The aforementioned remarks relate to the circuit level of analysis and suggest that CREB activation may specify circuits encoding the most relevant information in a learning experience. At the cellular level of analysis, two major issues concerning CREB are noteworthy, but not further investigated in this report. First, is CREB activation a correlate, necessity, cause, or effect of the experience-dependent representational change in the circuits that encode CTA and CCA? Second, to what extent does CREB activation itself contribute to the specificity of internal representations? The heavy issues of necessity and causality are of course not unique to our system, but it would be of interest to test the effect on CTA and CCA of in vivo modulation of CREB activation in the specific areas on which we focused [e.g., by the use of antisense (Guzowski and McGaugh, 1997; Lamprecht et al., 1997), RNA interference (Smalheiser et al., 2001), or conditional mutants (Mayford et al., 1996)]. As to the potential contribution of CREB to representational specificity, it is yet unsettled whether members of the CREB family are only switches that regulate universal operations in the activated circuits (i.e., the current predominant view), which is in line with the present results, or whether they also encode, in their mode of activation and the web of downstream intracellular events, specific information that 
contributes to the semantics of the relevant representation at the single-cell level (Egorov et al., 2002). Fine analysis of the effect of manipulation of CREB activation on long-term retention of specific items, using counterbalanced behavioral protocols that tap into different types of memory, might also contribute to the elucidation of this issue.

\section{References}

Bartsch D, Ghirardi M, Skehel PA, Karl KA, Herder SP, Chen M, Bailey CH, Kandel ER (1995) Aplysia CREB2 represses long-term facilitation: relief of repression converts transient facilitation into long-term functional and structural change. Cell 83:979-992.

Baxter MG, Chiba AA (1999) Cognitive functions of the basal forebrain. Curr Opin Neurobiol 9:178-183.

Berman DE, Hazvi S, Rosenblum K, Seger R, Dudai Y (1998) Specific and differential activation of mitogen-activated protein kinase cascades by unfamiliar taste in the insular cortex of the behaving rat. J Neurosci 18:10037-10044.

Berman DE, Hazvi S, Neduva V, Dudai Y (2000) The role of identified neurotransmitter systems in the response of insular cortex to unfamiliar taste: activation of ERK1-2 and formation of a memory trace. J Neurosci 20:7017-7023.

Bourtchuladze R, Frenguelli B, Blendy J, Cioffi D, Schutz G, Silva AJ (1994) Deficient long-term memory in mice with targeted mutation of the cAMP-responsive element-binding protein. Cell 79:59-68.

Brandner C, Schenk F (1998) Septal lesions impair the acquisition of a cued place navigation task: attentional or memory deficit? Neurobiol Learn Mem 69:106-125.

Brunzell DH, Kim JJ (2001) Fear conditioning to tone, but not to context, is attenuated by lesions of the insular cortex and posterior extension of the intralaminar complex in rats. Behav Neurosci 115:365-375.

Bucci DJ, Holland PC, Gallagher M (1998) Removal of cholinergic input to rat posterior parietal cortex disrupts incremental processing of conditioned stimuli. J Neurosci 18:8038-8046.

Bucci DJ, Conley M, Gallagher M (1999) Thalamic and basal forebrain cholinergic connections of the rat posterior parietal cortex. NeuroReport 10:941-945.

Bures J, Bermudez-Rattoni F, Yamamoto T (1998) Conditioned taste aversion: memory of a special kind. New York: Oxford UP.

Chiba AA, Bucci DJ, Holland PC, Gallagher M (1995) Basal forebrain cholinergic lesions disrupt increments but not decrements in conditioned stimulus processing. J Neurosci 15:7315-7322.

Chiba AA, Bushnell PJ, Oshiro WM, Gallagher M (1999) Selective removal of cholinergic neurons in the basal forebrain alters cued target detection. NeuroReport 10:3119-3123.

Dash PK, Hochner B, Kandel ER (1990) Injection of the cAMP-responsive element into the nucleus of Aplysia sensory neurons blocks long-term facilitation. Nature 345:718-721.

Desmedt A, Garcia R, Jaffard R (1998) Differential modulation of changes in hippocampal-septal excitability by the amygdala as a function of either elemental or contextual fear conditioning in mice. J Neurosci 18:480-487.

Desmedt A, Garcia R, Jaffard R (1999) Vasopressin release into the lateral septum promotes elemental conditioning to the detriment of contextual fear conditioning in mice. Eur J Neurosci 11:3913-3921.

Desmedt A, Garcia R, Jaffard R (2003) An 8-day extensive elemental, but not contextual, fear conditioning potentiates hippocampal-lateral septal synaptic efficacy in mice. Synapse 49:270-278.

Dudai Y (1989) The neurobiology of memory: concepts, findings, trends. Oxford: Oxford UP.

Dudai Y (1994) Molecular devices in neural learning machines, or the syntactic approach to biological learning. In: Memory system of the brain (Delacour J, ed), pp 319-336. Singapore: World Scientific.

Dudai Y (2002) Molecular bases of long-term memories: a question of persistence. Curr Opin Neurobiol 12:211-216.

Egorov AV, Hamam BM, Fransen E, Hasselmo ME, Alonso AA (2002) Graded persistent activity in entorhinal cortex neurons. Nature 420:173-178.

Frank DA, Greenberg ME (1994) CREB: a mediator of long-term memory from mollusks to mammals. Cell 79:5-8.

Garcia R, Jaffard R (1996) Changes in synaptic excitability in the lateral septum associated with contextual and auditory fear conditioning in mice. Eur J Neurosci 8:809-815.
Garcia J, Ervin FR, Koelling RA (1966) Learning with prolonged delay of reinforcement. Psychon Sci 5:121-122.

Giovannini MG, Mutolo D, Blanchi L, Michelassi A, Pepeu G (1994) NMDA receptor antagonists decrease GABA outflow from the septum and increase acetylcholine outflow from the hippocampus: a microdialysis study. J Neurosci 14:1358-1365.

Guzowski JF, McGaugh JL (1997) Antisense oligodeoxynucleotide-mediated disruption of hippocampal cAMP response element binding protein levels impairs consolidation of memory for water maze training. Proc Natl Acad Sci USA 94:2693-2698.

Hagan JJ, Salamone JD, Simpson J, Iversen SD, Morris RGM (1988) Place navigation in rats is impaired by lesions of medial septum and diagonal band but not nucleus basalis magnocellularis. Behav Brain Res 27:9-20.

Hall J, Thomas KL, Everitt BJ (2001) Fear conditioning retrieval induces CREB phosphorylation and Fos expression within the amygdala. Eur J Neurosci 13:1453-1458.

Hankins WG, Garcia J, Rusiniak KW (1974) Cortical lesions: flavor-illness and noise-shock conditioning. Behav Biol 10:173-181.

Impey S, Mark M, Villacres EC, Chavkin C, Storm DR (1996) Induction of CRE-mediated gene expression by stimuli that generate long-lasting LTP in area CA1 of the hippocampus. Neuron 16:973-982.

Impey S, Smith DM, Obrietan K, Donahue R, Wade C, Storm DR (1998) Stimulation of cAMP response element (CRE)-mediated transcription during contextual learning. Nat Neurosci 1:595-601.

Kelsey JE, Laudry BA (1988) Medial septal lesions disrupt spatial mapping ability in rats. Behav Neurosci 102:289-293.

Kida S, Josselyn SA, de Ortiz SP, Kogan JH, Chevere I, Masushige S, Silva AJ (2002) CREB required for the stability of new and reactivated fear memories. Nat Neurosci 5:348-355.

Kwok RP, Lundblad JR, Chrivia JC, Richards JP, Bachinger HP, Brennan RG, Roberts SG, Green MR, Goodman RH (1994) Nuclear protein CBP is a coactivator for the transcription factor CREB. Nature 370:223-226.

Lamprecht R (1999) CREB: a message to remember. Cell Mol Life Sci 55:554-563.

Lamprecht R, Hazvi S, Dudai Y (1997) cAMP response element-binding protein in the amygdala is required for long- but not short-term conditioned taste aversion memory. J Neurosci 17:8443-8450.

Marighetto A, Micheau J, Jaffard R (1994) Effects of intraseptally injected glutamatergic drugs on hippocampal sodium-dependent high-affinity choline uptake in "naive" and "trained" mice. Pharmacol Biochem Behav 49:689-699.

Marlin NA (1981) Contextual associations in trace conditioning. Anim Learn Behav 9:519-523.

Martin KC, Barad M, Kandel ER (2000) Local protein synthesis and its role in synapse-specific plasticity. Curr Opin Neurobiol 10:587-592.

Mayford M, Bach ME, Huang Y-Y, Wang L, Hawkins RD, Kandel ER (1996) Control of memory formation through regulated expression of a CaMKII transgene. Science 274:1678-1683.

McKinney M, Coyle JT, Hedreen JC (1983) Topographic analysis of the innervation of the rat neocortex and hippocampus by the basal forebrain cholinergic system. J Comp Neurol 217:103-121.

Meachum CL, Bernstein IL (1990) Conditioned responses to a taste conditioned stimulus paired with lithium chloride administration. Behav Neurosci 104:711-715.

Mesulam MM, Mufson EJ, Levey AI, Wainer BH (1983) Cholinergic innervation of cortex by the basal forebrain: cytochemistry and cortical connections of the septal area, diagonal band nuclei, nucleus basalis (substantia innominata), and hypothalamus in the rhesus monkey. J Comp Neurol 214:170-197.

Miranda MI, Ferreira G, Ramirez-Lugo L, Bermudez-Rattoni F (2002) Glutamatergic activity in the amygdala signals visceral input during taste memory formation. J Neurosci 99:11417-11422.

Miyamoto M, Kato J, Narumi S, Nagaoka A (1987) Characteristics of memory impairment following lesioning of the basal forebrain and medial septal nucleus in rats. Brain Res 419:19-31.

Muir JL, Everitt BJ, Robbins TW (1994) AMPA-induced excitotoxic lesions of the basal forebrain: a significant role for the cortical cholinergic system in attentional function. J Neurosci 14:2313-2326.

Nail-Boucherie K, Dourmap N, Jaffard R, Constentin J (2000) Contextual fear conditioning is associated with an increase of acetylcholine release in the hippocampus of rat. Brain Res Cogn Brain Res 9:193-197.

Nakamura N, Fujita H, Kawata M (2002) Effects of gonadectomy on immu- 
noreactivity for choline acetyltransferase in the cortex, hippocampus, and basal forebrain of adult male rats. Neuroscience 109:473-485.

Naor C, Dudai Y (1996) Transient impairment of cholinergic function in the rat insular cortex disrupt the encoding of taste in conditioned taste aversion. Behav Brain Res 79:61-67.

Olton DS, Wenk GL, Markowska AM (1991) Basal forebrain, memory and attention. In: Activation to acquisition: functional aspects of the basal forebrain cholinergic system (Richardson RT, ed), pp 247-262. Boston: Birkhäuser.

Otake K, Reis DJ, Ruggiero DA (1994) Afferents to the midline thalamus issue collaterals to the nucleus tractus solitarii: an anatomical basis for the thalamic and visceral reflex integration. J Neurosci 14:5694-5707.

Parker LA, Hutchison S, Riley AL (1982) Conditioned flavor aversions: a toxicity test of the anticholinesterase agent, physostigmine. Neurobehav Toxicol Teratol 4:93-98.

Paxinos G, Watson C (1986) The rat brain in stereotaxic coordinates. San Diego: Academic.

Radulovic J, Kammermeier J, Spiess J (1998) Relationship between fos production and classical conditioning: effects of novelty, latent inhibition and US pre-exposure. J Neurosci 18:7452-7461.

Rosenblum K, Meiri N, Dudai Y (1993) Taste memory: the role of protein synthesis in gustatory cortex. Behav Neural Biol 59:49-56.

Rosenblum K, Berman DE, Hazvi S, Lamprecht R, Dudai Y (1997) NMDA receptor and the tyrosine phosphorylation of its $2 \mathrm{~B}$ subunit in taste learning in the rat insular cortex. J Neurosci 17:5129-5135.

Schulz S, Siema H, Krug M, Höllt V (1999) Direct evidence for biphasic cAMP responsive element-binding protein phosphorylation during longterm potentiation in the rat dentate gyrus in vivo. J Neurosci 19:5683-5692.

Sheng M, McFadden G, Greenberg ME (1990) Membrane depolarisation and calcium induce $c$-fos transcription via phosphorylation of transcription factor CREB. Neuron 4:571-582.

Siegel JL (1976) Effect of medial septal lesions on conditioned taste aversion in the rat. Physiol Behav 17:761-765.

Silva AJ, Kogan JH, Frankland PW, Kida S (1998) CREB and memory. Annu Rev Neurosci 21:127-148.
Smalheiser NR, Manev H, Costa E (2001) RNAi and brain function: was McConnell on the right track? Trends Neurosci 24:216-218.

Sparks PD, LeDoux JE (1995) Septal lesions potentiate freezing behavior to contextual but not to phasic conditioned stimuli in rats. Behav Neurosci 109:184-188.

Spray KJ, Halsell CB, Bernstein IL (2000) c-Fos induction in response to saccharin after taste aversion learning depends on conditioning method. Brain Res 852:225-227.

Stanciu M, Radulovic J, Spiess J (2001) Phosphorylated cAMP response element-binding protein in the mouse brain after fear conditioning: relationship to Fos production. Mol Brain Res 94:15-24.

Swank MW (2000) Phosphorylation of MAP kinase and CREB in mouse cortex and amygdala during taste aversion learning. NeuroReport 11:1625-1630.

Taubenfeld SM, Wiig KA, Bear MF, Alberini CM (1999) A molecular correlate of memory and amnesia in the hippocampus. Nat Neurosci 2:309-310.

Thinus-Blanc C, Save E, Rossi-Arnaud C, Tozzi A, Ammassari-Teule M (1996) The differences shown by C57BL/6 and DBA/2 inbred mice in detecting spatial novelty are subserved by a different hippocampal and parietal cortex interplay. Behav Brain Res 80:33-40.

Vouimba RM, Garcia R, Jaffard R (1998) Opposite effects of lateral septal LTP and lateral septal lesions on contextual fear conditioning in mice. Behav Neurosci 112:875-884.

Wrenn CC, Lapi DA, Wiley RG (1999) Threshold relationship between lesion extent of the cholinergic basal forebrain in the rat and working memory impairment in the radial maze. Brain Res 847:284-298.

Yamamoto T (1994) A neural model for taste aversion learning. In: Olfaction and taste XI (Kurihara K, Suzuki N, Ogawa H, eds), pp 471-474. Tokyo: Springer.

Yamamoto T, Fujimoto Y (1991) Brain mechanisms of taste aversion learning in the rat. Brain Res Bull 27:403-406.

Yamamoto T, Shimura T, Sako N, Yasoshima Y, Sakai N (1994) Neural substrates for conditioned taste aversion in the rat. Behav Brain Res 65:123-137.

Yamamoto T, Fujimoto Y, Shimura T, Sakai N (1995) Conditioned taste aversion in rats with excitotoxic brain lesions. Neurosci Res 22:31-49. 